\title{
Análise in vitro, em mandíbulas humanas, da correlação da anti-língula com a língula em radiografias digitais
}

\section{Analyse in vitro, in humans mandibles, of antilingula with lingula correlation in digitals radiographs}

Luiz Roberto Coutinho MANHÃES Jr.

Especialista - Radiologia Odontológica - Faculdade de Odontologia de Piracicaba - UNICAMP - Mestrando - Programa de Pós-Graduação em Biopatologia Bucal - Faculdade de Odontologia de São José dos Campos - UNESP

\section{Cleber Frigi BISSOLI}

Mestrando - Programa de Pós-Graduação em Biopatologia Bucal - Faculdade de Odontologia de São José dos Campos - UNESP

\section{Horácio FAIG LEITE}

Professor Adjunto - Departamento de Biociências e Diagnóstico Bucal - Disciplina de Anatomia - Faculdade de Odontologia de São José dos Campos - UNESP

\section{Mari Eli Leonelli de MORAES}

Julio Cezar de Mello CASTILHO

Professor Assistente Doutor - Departamento de Cirurgia, Periodontia e Radiologia - Disciplina de Radiologia - Faculdade de Odontologia de São José dos Campos - UNESP

\begin{abstract}
Resumo
O trabalho visou analisar a correlação, em milímetros $(\mathrm{mm})$, do posicionamento da língula em relação à anti-língula (AL) em 143 mandíbulas maceradas de um total de 200, desconsiderando-se sexo e idade. Foram utilizadas radiografias periapicais digitais (sistema CCD) com a finalidade de se estabelecer estatisticamente a posição média desta relação anatômica. Para a mensuração da distância entre as estruturas foi usado o próprio software do aparelho digital. Encontrou-se uma média de 5,07mm para o lado esquerdo e 4,63mm para o direito. Houve correlação bilateral entre o posicionamento destas estruturas e em $71,50 \%$ foram possíveis à localização da AL.
\end{abstract}

\section{UNITERMOS}

Mandíbula, língula, anti-língula; radiografia digital

\section{INTRODUÇÃO}

Assim como qualquer outra especialidade da Odontologia, a Cirurgia evolui em grande escala. Técnicas cirúrgicas que eram realizadas por acesso externo, o que provocava seqüelas esteticamente deficitárias, hoje são realizadas por meio intrabucal, melhorando consideravelmente esse dano. Recursos tecnológicos avançados como meios de imagens mais modernos também favorecem esta realidade, podendo ser citada a tomografia computadorizada, imagem por ressonância magnética, programas que auxiliam nas mensurações e reconstruções em 3D. Levando-se em consideração os recursos tecnológicos existentes, cria-se uma esfera de opções aos cirurgiões no auxílio e planejamento das cirurgias ortognáticas.

Pacientes que apresentam prognatismo tanto maxilar quanto mandibular, ou seja, classificados como Classe II e III esquelética, possuem fortes indicações para sofrerem reduções através do método cirúrgico já que, nestes casos, apenas o tratamento ortodôntico não é o suficiente para se obter uma harmonia entre tecidos ósseos, tegumentares e estruturas dentárias. 
De acordo com a literatura ${ }^{8}$, a osteotomia sagital do ramo mandibular é certamente o procedimento mais usado em cirurgia ortognática. Também é indicada para a correção de prognatismo quando alterações verticais são previstas juntamente com o recuo antero-posterior. Na técnica cirúrgica a osteotomia é realizada paralela ao plano oclusal mandibular, em sentido posterior, logo acima e posterior a língula. Para a realização desta cirurgia, o Cirurgião Bucomaxilo-facial leva em consideração, como ponto de referência clínico, a estrutura anatômica denominada de anti-língula (AL) que fica situada na porção mais externa da face lateral do ramo mandibular. Está de acordo com Pogrel et al. ${ }^{7}$ (1995) que destacaram que a AL é uma estrutura anatômica importante para a execução da osteotomia do ramo mandibular. Esta estrutura não corresponde posicionalmente com a língula e muito menos com o forame mandibular. O fato do Cirurgião utilizá-la como orientação para o corte mandibular, não significa que seja confiável para a execução da técnica, pois pode afetar o nervo alveolar inferior. No entanto, esta só é visualizada no ato cirúrgico, sendo necessário ao profissional utilizar toda sua experiência clínica e noção da variabilidade anatômica existente para efetuar um procedimento mais seguro com o menor risco de dano ao paciente.

Muitos são os trabalhos que procuraram localizar a língula. Segundo Bremer ${ }^{1}$ (1952) quatrocentas mandíbulas foram estudadas para localizá-la em relação ao plano oclusal, encontrando-se $16 \%$ dos casos a 0 a $1 \mathrm{~mm}, 48 \%$ entre 1 a $5 \mathrm{~mm}, 27 \%$ de 5 a $9 \mathrm{~mm}, 5,2 \%$ entre 9 a $11 \mathrm{~mm}$ e a menor porcentagem $(4,8 \%)$ ficou para o posicionamento entre $11 \mathrm{a} 19 \mathrm{~mm}$, sendo todos acima do plano oclusal. Hayward et al. ${ }^{3}$ (1977) que avaliaram 45 crânios de asiáticos e 62 crânios de americanos para verificar a relação desta estrutura com o forame mandibular, observaram que a mesma encontrava-se mais anteriormente. Já para Kim et al. ${ }^{4}$ (1997) que estudaram 162 mandíbulas dentadas, realizando dois traçados retos, sendo um da borda anterior à borda posterior passando pela língula e outro da chanfradura sigmóide até a borda inferior também passando pela estrutura, a posição da língula em relação horizontal a borda do ramo mandibular foi em média de $17,4 \mathrm{~mm}$ para anterior. Entretanto, de acordo com Tuli et al. ${ }^{10}$ (2000) cujo trabalho foi avaliar as variações na forma anatômica da língula de 165 mandíbulas, sendo 134 de homens e 34 de mulheres, as formas obtidas foram $68,5 \%$ triangulares, $15,8 \%$ tronculares, $10,9 \%$ nodulares e $4,8 \%$ assimiladas.

Devido a estes fatos, o presente trabalho propõe verificar a presença, em milímetros $(\mathrm{mm})$, da correlação do posicionamento da língula em relação à $\mathrm{AL}$ em mandíbulas maceradas, utilizando radiografias digitais, a fim de se estabelecer estatisticamente à posição média dessa relação anatômica. Com esta mensuração, será possível criar um parâmetro para a atuação do profissional em seu campo de execução.

\section{Material e mÉtodo}

Inicialmente foram obtidas de um total de duzentas mandíbulas maceradas adultas do arquivo da Disciplina de Anatomia da Faculdade de Odontologia de São José dos Campos-Unesp, 143 mandíbulas nas quais a visualização da AL era possível.

As mandíbulas foram radiografadas com o aparelho 765 DC" da Gendex" (Chicago - IL - USA) sendo operado com $65 \mathrm{kVp}, 7 \mathrm{~mA}, 0,02 \mathrm{~s}$ de tempo de exposição e distância do foco-sensor de $40 \mathrm{~cm}$. Para a realização das radiografias periapicais utilizou-se o aparelho digital do sistema CCD (charge couple device) $R V G$ “ da Trophy" (Vallée - França) e seu respectivo software foi usado para a mensuração da distância entre a língula e a anti-língula. Para a identificação das estruturas, utilizou-se um pedaço de alumínio do tamanho da cabeça de um alfinete na AL e outro com forma de arco na língula o qual acompanhava seu contorno anatômico (Figuras 1 e 2).

Cada mandíbula foi radiografada 2 vezes, sendo uma do lado direito (D) e outra do lado esquerdo (E). O sensor foi posicionado paralelamente ao ramo mandibular que estava posicionado perpendicularmente ao feixe principal de Rx de tal forma que a área a ser radiografada estivesse na área útil do sensor.

Com as radiografias realizadas, foram feitas as mensurações da distância da língula à $\mathrm{AL}$ e analisadas estatisticamente pelos testes Anova e $t$ (Student). 


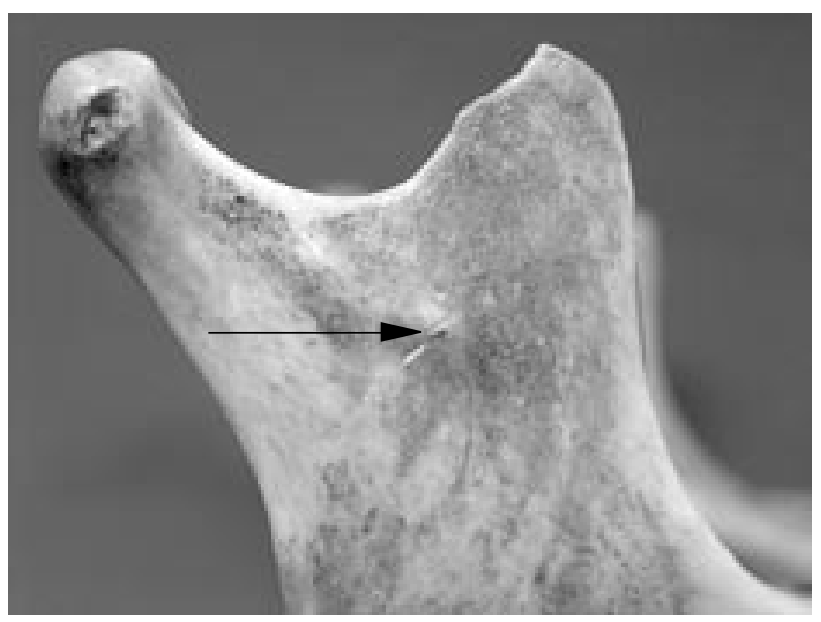

FIGURA 1 - Identificação da AL.

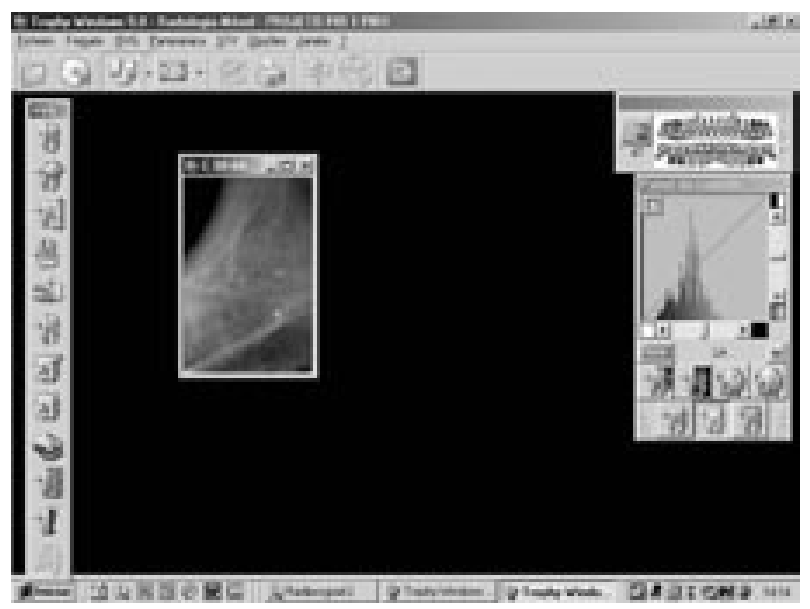

FIGURA 3 - Recorte da tela do computador ao se realizar a radiografia digital antes da mensuração.

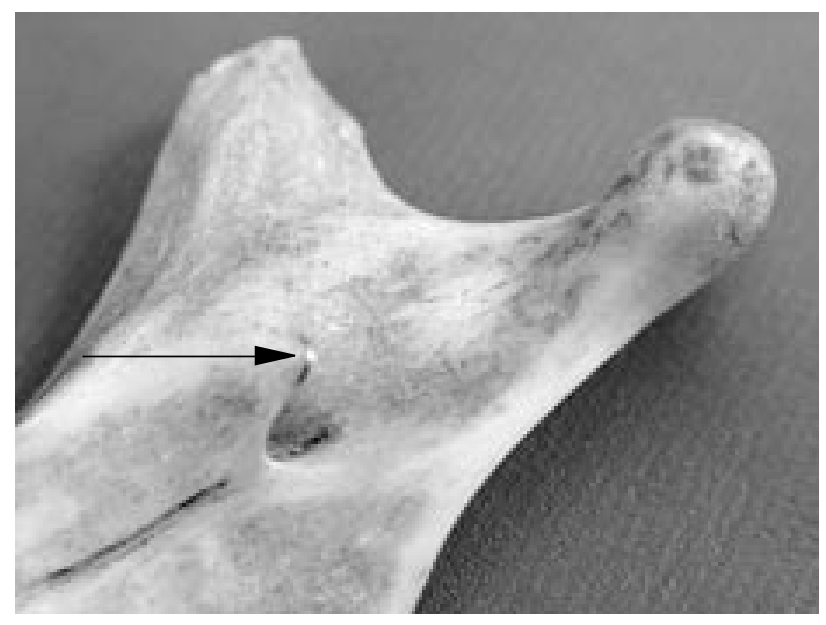

FIGURA 2 - Identificação da língula.

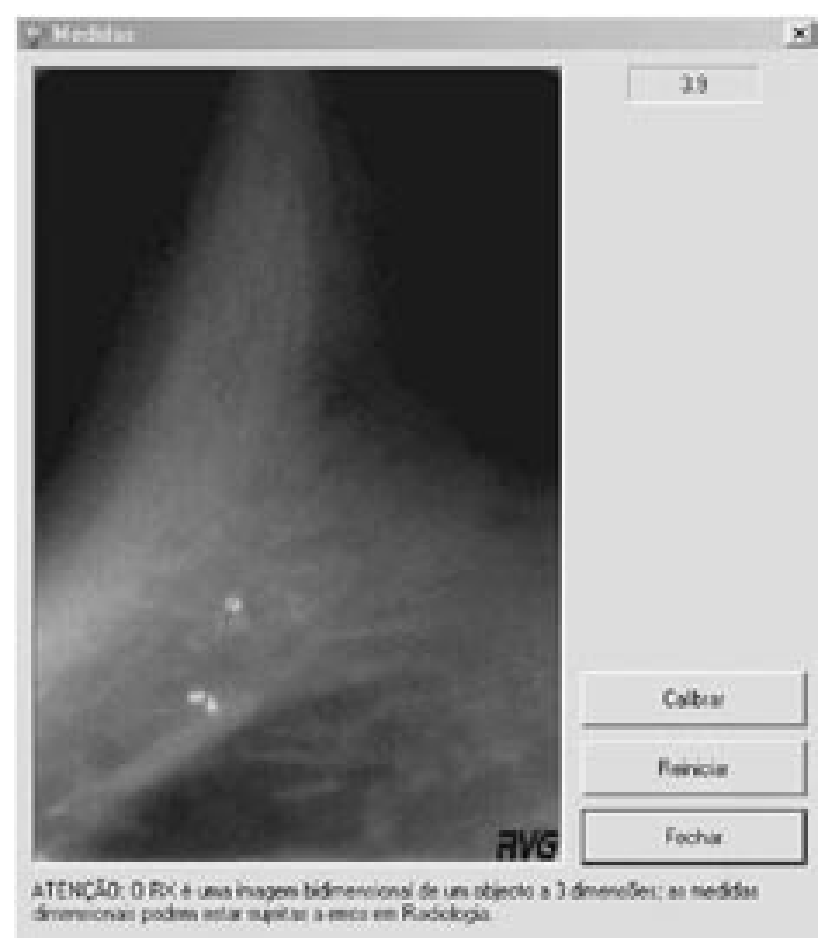

FIGURA 4 - Mensuração realizada do centro da imagem da AL à ponta do arco da língula. 


\section{Resultados}

Do total das duzentas mandíbulas estudadas, foi possível a localização da AL em 143 peças anatômicas, perfazendo 71,50\%. Após a análise estatística dos resultados pelo teste paramétrico Anova $(\mathrm{a}=5 \%)$ verificou-se que não houve diferença estatisticamente

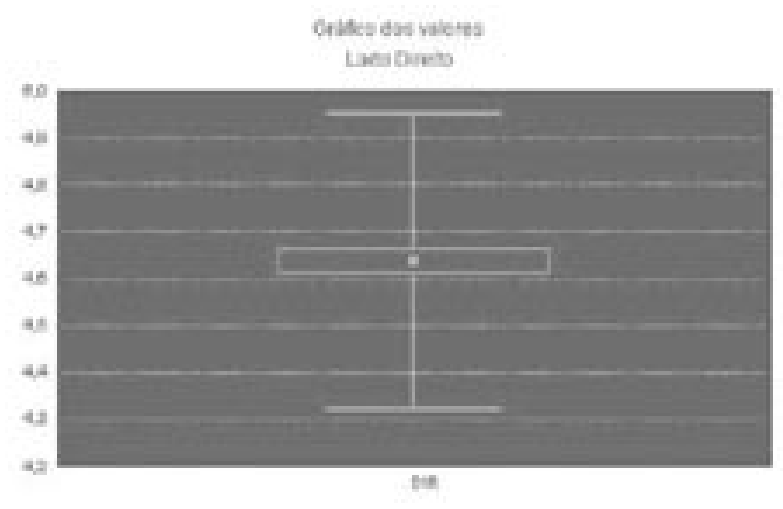

FIGURA 5 - Valores médios e desvio padrão do lado direito.

\section{Dıscussão}

Em cirurgia ortognática, na técnica da osteotomia em L-invertido, deve-se seguir uma linha cerca de 2 a $4 \mathrm{~mm}$ imediatamente superior e posterior a proeminência da AL, sendo considerado novamente sua importância ${ }^{2}$. Utilizou-se neste trabalho $71,50 \%$ das mandíbulas selecionadas das quais foram possíveis as localizações da AL, demonstrado na Figura 1, sendo muito próximas com as identificações de Yates et al. ${ }^{11}$ (1976) que estudaram setenta mandíbulas secas e visualizaram a AL em $72 \%$ da sua amostra. No entanto, contrapõe-se com Pogrel et al. ${ }^{7}$ (1995) que analisaram vinte mandíbulas de cadáveres humanos, utilizando três examinadores para a localização da AL encontrando-se apenas $22,50 \%$ e diferentes do estudo de Martone et al. ${ }^{6}$ (1993) no qual encontrou $42,86 \%$.

Assim como Martone et al. ${ }^{6}$ (1993) que avaliaram 63 hemi-mandíbulas de humanos, sendo 31 de homens e 32 de mulheres para verificar a distância entre a AL com o forame mandibular, Pogrel et al. ${ }^{7}$ significante $(\mathrm{p}=0,068)$ entre o lado $\mathrm{E}$ (média \pm desvio padrão: $5,07 \mathrm{~mm} \pm 2,09 \mathrm{~mm})$ e para o lado $\mathrm{D}(4,63 \mathrm{~mm}$ $\pm 1,90 \mathrm{~mm})$. Segundo o teste $t$, o intervalo de confiança para o $E$ foi de $4,72 \mathrm{~mm}$ a $5,41 \mathrm{~mm}$ e para o $\mathrm{D}$ foi de $4,32 \mathrm{~mm}$ a $4,95 \mathrm{~mm}$. Então, não houve diferença estatisticamente significante entre os lados E e D para o intervalo de confiança de $95 \%$.

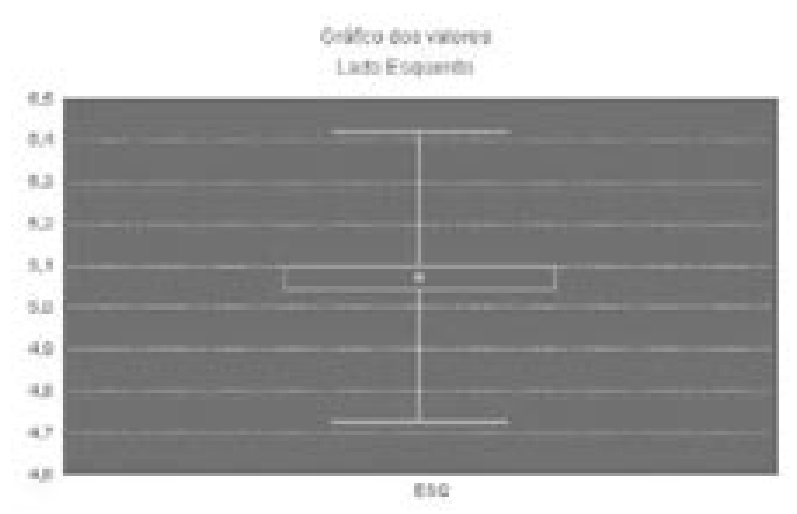

FIGURA 6 - Valores médios e desvio padrão para o lado esquerdo.

(1995) destacam a importância do posicionamento da AL para a execução da osteotomia do ramo mandibular em cirurgia ortognática o que justifica a escolha desta estrutura para a realização desta pesquisa, porém, a orientação não se torna confiável pelo fato da variabilidade anatômica. É claro que a criação de parâmetros médios possibilita o uso de mais uma ferramenta no auxílio aos Cirurgiões. Tratando-se dos valores médios encontrados (Figuras 5 e 6), há correspondência com os achados de Langstron \& Tebo $^{5}$ (1977) que relacionaram o forame mandibular com a proeminência lateral do ramo obtendo-se $4,7 \mathrm{~mm}$ de distância e com o estudo de Martone et al. ${ }^{6}$ (1993) que observaram a distância média entre a AL e o forame mandibular de $4,8 \mathrm{~mm}$, mas discrepante em relação a Yates et al. ${ }^{11}$ (1976) que encontraram a distância entre a AL e o forame mandibular em torno de 5 a $10 \mathrm{~mm}$ e com os $50 \%$ dos casos que estavam com uma diferença entre a AL e a língula de até $10 \mathrm{~mm}$ e $25,80 \%$ até $5 \mathrm{~mm}$ dos valores encontrado por Pogrel et al. ${ }^{7}$ (1995). 
Com relação à correlação entre os lados direito e esquerdo, há uma concordância com os estudos ${ }^{3}$ ${ }^{5}$ em que se verifica a correspondência estatisticamente significante, verificado nesta pesquisa pelo $p$ valor $\geq 0,05$. Com a tecnologia desenvolvida à área de imaginologia, a utilização do aparelho digital periapical possibilita a mensuração por meio de marcos (língula e AL) em softwares específicos, o que difere dos métodos utilizados nas pesquisas ${ }^{1,3,4}$ $5,6,10,11$ nas quais utilizam comparações entre língula e AL ao forame mandibular e borda lateral a língula. Apenas Pogrel et al. ${ }^{7}$ (1995) relacionam a localização da AL com a língula, porém sem a utilização dos aparelhos digitais, também sendo desconsiderado a idade e o sexo em decorrência de não diferirem estatisticamente $^{6}$.

\section{Conclusão}

Com a análise dos resultados, conclui-se que:

- Há correlação bilateral estatisticamente significante da posição da língula com a AL;

- A média da distância entre a AL e a língula no lado E é de $5,07 \mathrm{~mm}$ e para o D é de 4,63 $\mathrm{mm}$;

\begin{abstract}
The main aim of this research was analyzed, in millimeters $(\mathrm{mm})$, the correlation of the lingula e antilingula $(A L)$ positions in 143 dry mandibles of total 200, disrespecting sex and age. Digital periapical radiographs (system CCD) were used to establish the median position of this anatomic relation. To measurement of the distance between this structure was utilized the digital machine's own software. Found that the median of left side was 5,07mm and to right side 4,63mm. Bilateral correlation was noted between the position of these structures and 71,50\% were possible to localization the antilingula.
\end{abstract}

\title{
UNITERMS
}

Mandible lingula, antilingula; digital radiograph

\section{REFERÊNCIAS}

1. Bremer G. Measurements of special significance in connection with anesthesia of the inferior alveolar nerve. Oral Surg 1952; 5: 966-88.

2. Hall DH. Mandibular Prognatism. In: Bell WH. Modern Practice in Orthognathic and Reconstructive Surgery. Philadelphia: Saunders Company; 1992. p.2111-69

3. Hayward BS, Richardson ER, Malhotra SK. The mandibular foramen Its anteroposterior position. Oral Surg.Oral Med. Oral Pathol, 1977 Dec.; 44(6): 837-43.

4. Kim HJ, Lee HY, Chung IH, Cha IH, Yi CK. Mandibular anatomy related to sagittal split ramus osteotomy in koreans. Yonsei Medical J; 1997; 38(1): 19-25.

5. Langstron JR; Tebo HG. The incidence and relationship of the lateral ramus prominence to the mandibular foramen. Oral Surg Oral Med Oral Pathol; 1977 Aug.; 44(2): 190-6.

6. Martone CH, Bem-Josef AM, Wolf S, Mintz SM. Dimorphic study of surgical anatomic landmarks of the lateral ramus of the mandible. Oral Surg Oral Med Oral Pathol; 1993 Apr.; 75(4): 436-8.

7. Pogrel MA, Schmidt BL, Ammar A. The presence of the antilingula and its relationship to the true lingula. Br J Oral Maxillofac Surgery; 1995 Aug.; 33(4): 235-8.
8. Polido WD. Osteotomia sagital do ramo mandibular. In : Araújo A. Cirurgia Ortognática. São Paulo: Ed. Santos;1999. p. 113-30.

9. Reitzik M, Griffiths RR, Mirels H. Surgical anatomy of the ascending ramus of the mandible. Br J Oral Surg.1976; 14(2): 150-5.

10. Tuli A, Choudhry S, Raheja S, Agarwal S. Variation in shape of the lingula in the adult human mandible. J Anat 2000 Aug.; 197(pt 2): 313-17.

11. Yates C, Olson D, Guralnick W. The antilingula as an anatomic landmark in oral surgery. Oral Surg.1976 June; 41(6): 705-8.

Recebido em: 30/11/03 Aprovado em: 09/02/04

Luiz Roberto Coutinho Manhães Jr. Faculdade de Odontologia de São José dos Campos - UNESP Disciplina de Radiologia Av: Francisco José Longo, 777 Jardim São Dimas - Cep: 12450-000 Tel: (12) 3947-9054 / Fax: (12) 3947-9010. e-mail: lrmanhaesjr@fosjc.unesp.br 